\title{
Retrait thérapeutique: la place de l'autonomie
}

\author{
A. L. Dalle Ave ${ }^{a, b}$, Samia Hurst ${ }^{b}$ \\ ${ }^{a} \mathrm{MD}$, Unité d'éthique, CHUV; ${ }^{\mathrm{b}} \mathrm{MD}$, Institut Ethique Histoire Humanités, Genève
}

Entscheid zum Therapieabbruch: der Stellenwert der Selbstbestimmung Der Entscheid zum Therapieabbruch kann zu einem ethischen Dilemma führen, bei dem sich grundlegende moralische Werte gegenüberstehen. Ausgehend von einem konkreten klinischen Fall wird im Folgenden der Stellenwert der Selbstbestimmung bei einem solchen Entscheid analysiert. Dabei soll aufgezeigt werden, wie die Berücksichtigung der Selbstbestimmung mit den anderen Werten verknüpft sein kann. Ausserdem sollen die Grenzen des Begriffs Nutzlosigkeit ausgelotet werden.

\section{Introduction}

La médecine doit s'exercer dans le respect des droits du patient et des obligations des professionnels de la santé (cf. Tab. 1) [1]

Cependant, lorsque les avis divergent face à un dilemme éthique, comment décider quelle voie adopter? Par exemple, le patient a-t-il le droit d'exiger des soins jugés futiles par les professionnels? Qu'entend-on au juste par futilité?

Le Code civil suisse, repris dans les recommandations de l'Académie suisse des sciences médicales (ASSM), prévoit que le respect de la volonté du patient atteint ses limites quand un patient réclame des mesures qui sont inefficaces ou inappropriées [2]. Une action est efficace, si elle génère l'effet escompté. Ce qui rend une action appropriée est moins bien défini et peut être source de dilemmes décisionnels dans la pratique clinique. Nous décrirons ici la situation d'un patient qui, malgré l'avis médical de pratiquer un retrait thérapeutique, persiste dans sa volonté de vivre. Par le biais de cette situation clinique, nous établirons les étapes im- portantes de l'analyse éthique des situations médicales difficiles et explorerons les limites du concept de futilité.

\section{Cas clinique}

Le cas clinique décrit ci-dessous est basé sur une histoire vraie. Il a été modifié à des fins de confidentialité.

$M$. Vie est un patient de 75 ans, qui, suite à une greffe pulmonaire, a développé une infection opportuniste $\mathrm{X}$, avec pneumonite et hépatite. Il est actuellement hospitalisé aux soins intensifs, intubé, et requiert un soutien ventilatoire modéré. La dernière semaine s'est compliquée avec une aggravation des tests hépatiques et des fonctions respiratoires. L'infection X est résistante au traitement usuel, et malgré un traitement combiné, la situation médicale s'empire.

Les médecins en charge du patient, devant cette situation médicale dépassée, optent pour un retrait thérapeutique. Le médecin assistant est choqué par cette décision, car le patient lui aurait exprimé la volonté de vivre. Après une discussion initiale au sein de l'équipe, l'unité d'éthique de son hôpital est contactée.

\section{Analyse éthique de la situation médicale}

Pour aborder les situations éthiquement difficiles, il est utile de procéder à une analyse par étapes (cf. Tab. 2) [3].

\section{Reconnaître l'existence d'un dilemme éthique}

La première étape de toute analyse éthique est de re-

Tableau 1: Droits du patient et obligations des professionnels.

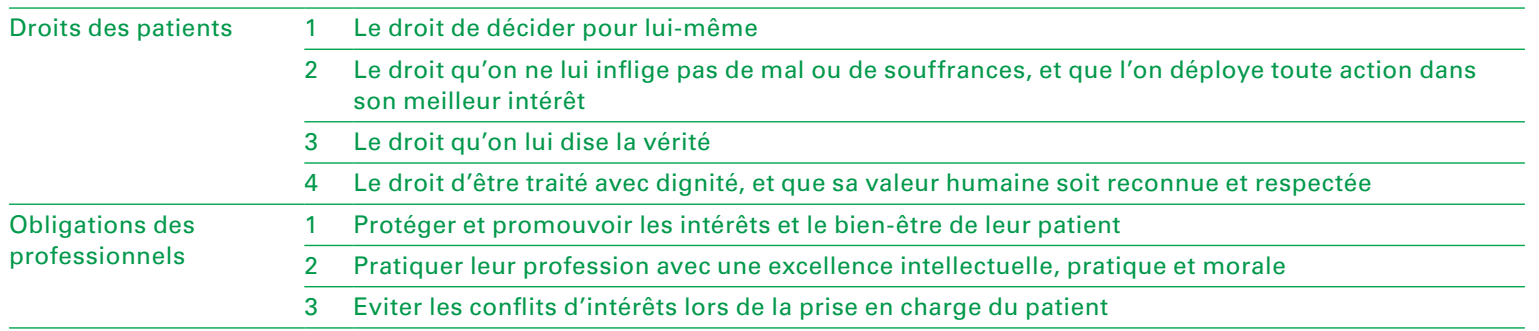


Tableau 2: Analyse éthique par étape.

\begin{tabular}{ll}
$\frac{1}{2}$ & Reconnaître l'existence d'un dilemme éthique \\
\hline 3 & Expliquer le problème éthique \\
\hline 4 & Eclaircir la situation médicale et le pronostic du patient \\
\hline 5 & $\begin{array}{l}\text { Analyser les notions principales (ici la notion de futilité) } \\
\text { utilisées pour justifier les positions en présence }\end{array}$ \\
\hline 6 & Explorer les alternatives possibles
\end{tabular}

connaître que la situation médicale engendre un problème éthique [3]. Effectivement, les professionnels de la santé qui ne reconnaissent pas les dilemmes éthiques dans leur pratique quotidienne risquent de prendre des décisions médicales basées sur des considérations incomplètes, parfois au dépend du bien de leurs patients.

\section{Expliquer le problème éthique}

Dans le cas qui nous intéresse, nous pourrions expliquer le problème éthique ainsi: Est-ce que les traitements qui maintiennent le patient en vie devraient être retirés, au nom du principe de bienfaisance, en sachant que la conséquence sera le décès rapide de $M$. Vie? Ou bien est-ce qu'ils devraient être poursuivis selon la volonté du patient, pour respecter le principe d'autonomie?

\section{Eclaircir la situation médicale et le pronostic du patient}

L'analyse éthique ne pourra être fondée que si la compréhension de la situation médicale est complète.

Pour M. Vie, les médecins en charge des soins intensifs confirmeront que la situation médicale est dépassée, qu'elle échappe au traitement, et qu'il n'existe aucun moyen de guérir le patient. En gardant la thérapie actuelle maximale, ils évaluent l'espérance de vie du patient à trois mois. Les spécialistes contactés, dont le pneumologue et l'infectiologue, sont moins catégoriques et rapportent de rares cas où des patients en situation semblable auraient survécu plus longtemps. Ce scénario met en évidence que la médecine n'est pas une science exacte, particulièrement quant à l'évaluation du pronostic d'un patient. Tenir compte de cette incertitude permettra un avis médical plus proche de la réalité.

\section{Evaluer la situation sous l'angle des valeurs présentes}

Cette étape consiste à identifier les différentes valeurs en tension dans la situation, dont les principes d'autonomie, de bienfaisance et de justice distributive. La bienfaisance est l'engagement des professionnels à pro- mouvoir le bien-être de leur patient [1]. L'autonomie est l'implication des valeurs et des souhaits du patient dans les décisions médicales [1]. La justice distributive est l'allocation équitable des biens et ressources [3]. Ces différentes valeurs (ou principes) ne sont pas absolues et leurs applications pratiques nécessitent une adaptation en fonction des cas concrets.

\section{Principe d'autonomie}

Le principe d'autonomie se compose de plusieurs éléments qu'il conviendra d'évaluer afin de déterminer s'ils sont présents ou non.

1. Le médecin est responsable de fournir une information adéquate, loyale, et adaptée au patient de façon transparente en évitant au mieux les conflits d'intérêts.

2. Le patient a-t-il compris l'information? Lors de cette étape, il faut déterminer si le patient base son choix sur une information correctement comprise.

3. Le patient a-t-il la capacité de choisir, a-t-il sa capacité de discernement? Il y a différents outils pour évaluer la capacité de discernement des patients [4]. Si le recours à un psychiatre peut être utile en cas de doute sur l'impact d'une maladie mentale sur la capacité de discernement, cette évaluation peut être réalisée dans la plupart des cas par tout médecin. Si le patient n'est pas capable de discernement, il faudra tenter de reconstruire sa volonté présumée, en se renseignant auprès de sa famille ou en se référant à d'éventuelles directives anticipées.

Dans le cas de M. Vie, nous admettrons pour l'exercice que le patient a sa capacité de discernement, même si sa stricte évaluation pourrait bien être ardue chez ce patient intubé. Ainsi, le patient exprime clairement son souhait de vivre, il ne veut pas être extubé, il ne veut pas mourir, et souhaite que tous les traitements soient continués tels quels. Il a bien compris que son pronostic vital est réservé, que la situation médicale va sans doute s'aggraver et qu'il ne pourra sans doute pas sortir des soins intensifs vivant.

4. Quelles sont les préférences et les valeurs du patient motivant son choix? Qu'espère-t-il en matière de qualité de vie? Il s'agit de mieux comprendre si ce que le patient vise est réaliste, et comment la priorité actuelle du patient s'inscrit dans ses choix de vie [5]. En discutant plus en avant avec M. Vie, ce dernier exprime un élément nouveau: il souhaite que les traitements soient maintenus tant qu'il pourra interagir avec son environnement. Dès le moment où il perdrait conscience de manière irréversible, il souhaite que les thérapies qui le maintiennent en vie soient retirées. 
La littérature en expose trois versions principales [1]:

1. la futilité physiologique: un traitement futile n'apporte aucun effet physiologique, et ne permet pas le maintien des fonctions vitales;

2. la futilité quantitative, basée sur l'évaluation du pronostic vital du patient: par exemple, un traitement futile permet de rétablir ou de maintenir les fonctions vitales chez moins de $1 \%$ des patients présentant un tableau clinique similaire;

3. la futilité qualitative, basée sur l'évaluation de la qualité de vie du patient: une intervention est futile, si elle ne permet pas le retour à une certaine qualité de vie, intégrant des notions variables, allant de l'indépendance du patient dans les activités quotidiennes à sa capacité d'interagir avec son environnement.

Ces définitions recouvrent des réalités qui peuvent être très différentes. En cas d'inefficacité ou de futilité physiologique, les professionnels ne devraient pas proposer une procédure diagnostique ou thérapeutique. En dehors de la claire futilité physiologique, cependant, la notion de futilité est rarement suffisante comme raison au retrait thérapeutique. La futilité quantitative et qualitative requièrent en plus un consensus autour du seuil à appliquer.

Le cas de M. Vie n'entre dans aucune des définitions de la futilité présentées ici. La continuation des traitements en cours permettrait un maintien des fonctions vitales chez plus de $1 \%$ des patients et permettrait au patient une certaine interaction avec son environnement. La futilité ne serait donc ici pas une justification raisonnable au retrait thérapeutique.

\section{Explorer les alternatives possibles}

L'analyse éthique a permis de révéler qu'il existait d'autres alternatives thérapeutiques qui permettraient de trouver un consensus entre les différentes visions du bien exposées.

Il serait possible, selon les souhaits du patient, de maintenir les traitements, jusqu'à ce que ce dernier perde de façon irréversible la possibilité d'interagir avec son environnement.

Si des questions de justice distributive sont en jeux, il faudrait évaluer la possibilité de continuer les traitements dans une structure moins aiguë que celle des soins intensifs, en acceptant que ce soit une alternative moins sécuritaire, et moins habituelle.

\section{Conclusion: L'essentiel pour la pratique}

Cette situation met en évidence une des tensions que nous retrouvons souvent dans nos consultations d'éthique, entre ce que souhaite le patient (ou ses proches) et ce que souhaitent les différents professionnels de la santé.

Afin d'établir quelle voie est la plus proche des principes éthiques, il est nécessaire de décortiquer la situation clinique étapes par étapes. La vision que le patient a de son propre bien doit être bien explorée, afin de mieux comprendre son choix et comment il s'inscrit dans ses priorités.

Il importe aussi d'apporter un soin particulier à la protection des patients en situation de vulnérabilité, surtout si, comme dans le cas de M. Vie, ils n'ont pas de proches pour défendre leurs intérêts.

En réalité, dans le cas de $M$. Vie, l'unité d'éthique n'a pas été sollicitée et cette analyse n'a pas eu lieu. Les mesures thérapeutiques ont été retirées, et le patient est décédé le lendemain. Ce cas clinique illustre un cas où il est probable que l'autonomie du patient n'a pas été suffisamment prise en compte. Il illustre la difficulté qu'il peut y avoir à faire face à un problème éthique sans en maîtriser suffisamment les enjeux. La discussion en équipe interprofessionnelle est ici primordiale. Les unités d'éthique, à disposition de tout professionnel de la santé en charge des patients, offrent ainsi une plateforme neutre de discussion, qui conseillent les cliniciens dans la prise de décisions médicales, dans le respect des valeurs éthiques.

\section{Références}

1 Bruce CR, Majumder MA, Bibler T, et al. A practical guide to developing and sustaining a clinical ethics consultation service. Baylor College of Medicine 2015.

2 ASSM. Bases juridiques pour le quotidien du médecin. $2^{\mathrm{e}}$ édition, 2013.

3 Hébert PC. Doing Right. A practical guide to ethics for medical trainees and physicians. 3nd Edition 2014. Oxford University Press.

4 Appelbaum PS. Clinical practice. Assessment of patients' competence to consent to treatment. N Engl J Med 2007;1,357(18): 1834-40.

5 Brody H, Clark M. Narrative Ethics: A Narrative. Hastings Center Report. 2014 Jan;44:S7-S11. PubMed PMID: WOS:000330629000003. English.

\section{Disclosure statement}

Aucun conflit d'intérêt.

\section{Mots clefs}

retrait thérapeutique, éthique, autonomie, futilité, bienfaisance, justice distributive 